\title{
2. Ways of knowing with data visualizations
}

\author{
Jill Walker Rettberg
}

\begin{abstract}
Data visualizations combine numeric data with visual representation, and these modes allow them to express certain kinds of knowledge more easily than others. This chapter uses examples of historical data visualizations in order to examine what ways of knowing they privilege. What is the difference between the spatial organization of tools in prehistoric homes and a photograph or bar chart showing information about the same tools, in terms of the kinds of knowledge they enable? How do the systems for gathering and visualizing data during the $18^{\text {th }}$ and $19^{\text {th }}$ centuries shape our understanding of the world? How do data visualizations make us feel that they are objective? How do they shape our ideas of what is possible?
\end{abstract}

Keywords: Dataism; God trick; Desire for numbers; Correlation and causation; The sublime; Epistemology of data visualization

\section{Introduction}

Data visualizations combine at least two modes of representation: numerical data and visual diagrams. For a computer program to be able to process data, it has to be converted to numbers, to the zeros and ones of machine code. In addition, the data need to be visually organized, which often requires dividing them into discrete quantities where lines, size, spatial placement, and other visual elements show certain patterns in the data. Each of these two modes of expression, the numeric and the visual, carries its own affordances and constraints for what they can express.

This anthology has several chapters that use concrete examples to discuss how data visualizations can be biased in their representations of

Engebretsen, M. and H. Kennedy (eds.), Data Visualization in Society. Amsterdam: Amsterdam University Press, 2020 DOI 10.5117/9789463722902_CHO2 
data (Ricker, Kraak, \& Engelhardt, this volume; D'Ignazio \& Bhargava, this volume) or how data visualizations can work against the typical abstraction they entail to include individuals' stories (Alamalhodaei, Alberda, \& Feigenbaum, this volume). My emphasis in this chapter is on examining the underlying mechanisms of data visualizations as an assemblage of data and visualizations. My exploration sits alongside existing critical work on data visualizations in feminist scholarship (D'Ignazio \& Klein, 2016; Hill, Kennedy, \& Gerrard, 2016), in the digital humanities (Drucker, 2011, 2014; Gitelman, 2013), and in critical algorithm studies and other scholarship on the epistemological basis for algorithmic processing of big data (Eubanks, 2018; Gillespie \& Seaver, 2015; Noble, 2018).

\section{Visual organization}

Organizing objects visually and spatially is something humans and our ancestors have done for a long time. In her essay 'Visualizing Thought', Barbara Tversky describes how hominins living three-quarters of a million years ago organized their tools and belongings in different areas of their home. She argues that this is the basic precursor to any kind of visualization: 'Perhaps the simplest way to use space to communicate is to arrange or rearrange things in it. An early process is grouping things in space using proximity, putting similar things in close proximity and farther from dissimilar things' (Tversky, 2010, p. 504). We might extend Tversky's line of reasoning to the modern domestic habit of keeping forks in one partition of a kitchen drawer and knives in another, and argue that this is a way of visually and spatially communicating information about the forks and knives.

The data visualizations we see on computer screens or printed pages, or even early markings on stones or in the sand, are one step removed from the phenomena they represent or organize. If we walk into somebody's kitchen and open a drawer, we see the knives and forks in the kitchen drawer, but we also experience them in space, and we can touch them and pick them up. Now, imagine a data visualization about kitchen utensils on a screen. It could be very simple, showing the number of knives and forks and other utensils in a kitchen, perhaps organized as a bar chart, perhaps using little pictures of forks stacked up in one bar and knives in another to show the relative quantities. Or imagine a photograph of the kitchen drawer, or an Instagram-style flat lay photograph of all the knives and forks neatly laid out on a table and photographed from above. 
Once the knives and forks are transferred from spatially organized objects to a visual representation on a two-dimensional surface, our distance from them increases. We interpret them as separate from us. A photograph of the drawer might not encourage a great deal of analytical dissection of the image, but the neatly organized flat lay photograph and the bar graph prioritize an analytic approach to that which is represented.

In his influential book about the transition from oral to literate cultures, Walter Ong (1982) argues that a fundamental difference between orality and literacy is that the visual nature of writing leads to ideas of objectivity that are impossible in oral culture. When we speak to each other in a face-to-face conversation, we are immersed in the sound, and because the speakers are in the same physical space, face-to-face oral discourse tends to be situated and concrete. Writing, on the other hand, separates the knower from the known. There is a distance between reader and writer. 'Sight isolates', Ong writes, while 'sound incorporates. Whereas sight situates the observer outside what he views, at a distance, sound pours into the hearer' (1982, p. 45). A typical visual ideal is clarity and distinctness, a taking apart, Ong argues, whereas the auditory ideal, by contrast, is harmony, a putting together (p. 71). He writes: 'A sound-dominated verbal economy is consonant with aggregative (harmonizing) tendencies rather than with analytic, dissecting tendencies (which would come with the inscribed, visualized word: vision is a dissecting sense)' (p. 73).

Ong does not discuss visualizations or diagrams, but following his reasoning, we can see a similar transition from the spatial organization of objects to the visual representation of objects on a page or other flat surface. Think back to the drawer of knives and forks as a way of organizing data: when there are real knives and real forks, the human is able to pick up a knife or a fork, move them around, manipulate them. Touch, like sound, involves closeness and participation. But the moment we switch from a physical drawer to a visual representation of a drawer, we are placed outside the representation, as analytical observers who feel an objective distance from what is seen. At least, this is true if we follow Ong, and not all would: Jonathan Sterne, for instance, criticizes Ong's framework as too simple a binary, too closely based on theological distinctions about the meaning of 'the word', and too little grounded in existing anthropological research on oral and literate cultures (Sterne, 2011). But whether or not Ong's framework is too simplistic, the basic idea that visual representation can lead to a more analytic approach is also expressed by other scholars coming from very different angles. Tversky also emphasizes analysis as key in visualization, but for her it is the persistence of images, that is, that they are not fleeting 
like the spoken word, that allows the 'perceptual processes' to occur that are needed for 'understanding, inference, and insight':

Because [images] persist, they can be subjected to myriad perceptual processes: Compare, contrast, assess similarity, distance, direction, shape, and size, reverse figure and ground, rotate, group and regroup; that is, they can be mentally assessed and rearranged in multiple ways that contribute to understanding, inference, and insight. (Tversky, 2010, p. 500)

\section{Systematizing data}

Importantly, not only the visual, but also the data themselves share much of this promise of analytical objectivity. Data visualization had a golden age in the nineteenth century, at the same time as nation states began largescale collection of statistical data (Friendly, 2006). However, it began a few centuries earlier, at the same time as the scientific method was developing, and with it the idea that humans could precisely observe the world and use those observations to understand it. Seventeenth- and eighteenth-century Europe saw an increasing trend towards observation, measurement, and quantification, and different fields developed new ways of measuring and quantifying things that had not previously been seen as interesting. Some of these methods were technological. For instance the invention of the telescope allowed Galileo to make observations about the solar system that would not previously have been possible. In our time, the existence of precise sensors and of computers that can process massive amounts of data allows for certain types of measurement, analysis, and visualization that were not possible a few decades ago.

Social and organizational changes also led to new kinds of quantification. National registries became common during the nineteenth century, for instance, allowing for analysis of trends over time or the comparison of different regions. For example, the first centralized national system of crime reporting was instituted in France in 1825, and collected information about all charges made in French courts on a quarterly basis (Friendly, 2006, p. 25). More and more information was collected, and by the end of the nineteenth century the French police not only had detailed statistics about crimes, but also systems for documenting and identifying criminals and suspects using a system of 'anthropometrics', devised by Alphonse Bertillon and involving very specific measurements of body parts (Kember, 2014). Once one has such a system, once it is possible to gather data that appears 
to give us knowledge, we end up with what Helen Kennedy calls a 'desire for numbers' that can lead to a lack of critical reflection about what those numbers mean and whether we truly need them (2016, p. 51).

This sense that systematized data have authority is an important aspect of the rhetorical power of data visualizations. While Ong and Tversky emphasized the visual as allowing for an analytical and perhaps objective stance, many have argued that it is the data themselves, or the quantitative nature of data visualizations, that lend them this sense of authority.

\section{A perception of objectivity}

According to Anthony McCosker and Rowan Wilken (2014), data visualizations often offer a 'fantasy of knowing' or of 'total knowledge', or in Donna Haraway's words, they claim to present a 'God's eye view' (Haraway, 1988, p. $5^{81)}$. The use of a data visualization in a newspaper article or a corporate report carries with it a rhetorical weight: the simple presence of the data visualization seems to state 'Look, we have data. This is true' (see Tal, Aner, \& Wansink, 2016 on data visualization's association with truthfulness).

José van Dijck uses the term dataism to describe the ideology of big data, which is characterized by 'a widespread belief in the objective quantification and potential tracking of all kinds of human behavior and sociality through online media technologies' (2014, p. 198). Epistemologically, data visualizations build upon this trust in data.

We can trace many histories of society's growing trust in numbers. The registration of data about crimes and criminals mentioned above tells of one such history, which can be traced forwards to today's bodycams, surveillance, and biometrics (Gates, 2011). Another, parallel history is that of the transition from midwives and their home-based care of mothers and infants to the increasing medicalization of prenatal care. This story can be told as a transfer of power from women to men, but it can also be seen as a transfer of trust from humans to machines, as the increasing institutionalization of prenatal and infant care included a radical growth in the use of technology to monitor growth and health (Oppenheimer, 2013). Today, iPhone apps connect to digital scales that generate daily data visualizations of a baby's weight (Rettberg, 2014, p. 67) and smart socks generate continuous visualizations of a baby's heartbeat (Leaver, 2017).

The management of birth is one thread in this story of numbers. Another thread is the management, or perhaps rather the marketing, of instruments of death, as told by Donald Mackenzie in Inventing Accuracy: A Historical 
Sociology of Nuclear Missile Guidance (1993). Or we might consider the prevention of life, a thread of the story told by Michelle Murphy in The Economization of Life (2017), where she discusses how demographic models comparing population size and financial growth created programmes intended to improve the future economies of developing countries through extensive birth control and abortion programmes.

\section{The average as norm}

Displaying data visually rather than as a table of numbers is a powerful method for finding patterns in the data. Some patterns recur in many different datasets, such as the bell-shaped curve seen in Figure 2.1, a graph showing the heights of Belgian men, which follows what is mathematically known as a normal distribution. Writing in the 186os, Adolphe Quetelet interpreted this recurrence as evidence of a fundamental social law, and defined the central portion of the curve as 'normal', with those outside the normal zone seen as aberrations (1997). Sekula explains that ' $t$ t]hus conceived, the "average man" constituted an ideal, not only of social health, but of social stability and of beauty' (1986, p. 22). Quetelet's work leaned heavily upon data visualizations. He first showed his data in the form of a table, then showed it visualized, drawing conclusions from the patterns that became apparent when the numbers were shown as curves on an $\mathrm{x}$ - and $\mathrm{y}$-axis.

The power of visualizations to show averages and patterns contributed to the nineteenth-century privileging of the 'norm', or as Lennard Davis argues, a 'generalized notion of the normal as an imperative', where 'the average then paradoxically becomes a kind of ideal, a position to be wished' (Davis, 2013, p. 2). This privileging of the average is a marked break from earlier traditions that saw the ideal body, represented for instance in paintings of Venus, as something 'mytho-poetical', a 'divine body' that is 'not attainable by a human' (Davis, 2013, p. 2).

As it turns out, the average human doesn't exist. Yes, that even curve shape shown in Figure 2.1 does show up again and again when you measure almost any aspect of humans - or of most things, really. But that doesn't mean that any individual human is 'average'. In her book Technically Wrong (2017), Sara Wachter-Boettcher tells the story of how the adjustable seatbelt was designed. Prior to its invention, the air force planned to design cockpits that fit 'the average pilot' - but they discovered that none of their pilots were of average size in all the ten dimensions they measured, such as height, 


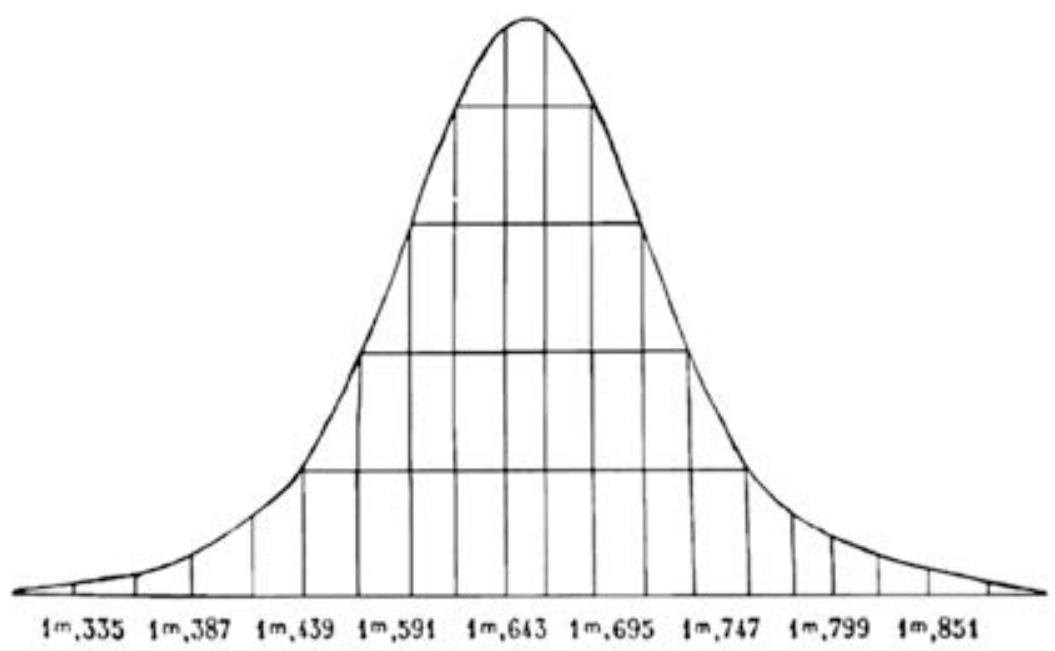

Figure 2.1. The height of Belgians from 18 to 20 years. Reprinted from Physique sociale ou Essai sur le développement des facultés de l'homme (p. 355), by A. Quetelet, 1997 [1869], Brussels: Académie Royale de Belgique. Copyright 1997 by Académie Royale de Belgique. Reprinted with permission.

wrist circumference, and shoulder width. Wachter-Boettcher uses this point to argue that it's important to design technology that fits people at each extreme rather than for the average person, as the air force did by creating adjustable seats and seat belts (Wachter-Boettcher, 2017). The idea of 'the average' may be encouraged by data visualizations, but that doesn't mean that it's necessarily the most useful way of viewing the data.

\section{Correlation is easier than causation}

Computers are extremely good at finding correlations. In fact, this is one of the mainstays of current models of deep machine learning, where software is fed 'big data' and works through it to find patterns. By analysing historical data, computers can find patterns that allow them to predict future behaviour. Often these predictions are eerily accurate. In some tests, AI systems do a better job at medical diagnosis than human doctors (Olson, 2018). It is wise to remember, though, that many stakeholders have a strong financial interest in convincing the general public that $\mathrm{AI}$ is efficient, perhaps more efficient than humans, and AI's ability to make accurate predictions is often overstated.

Visualizations of data also prioritize correlation over causation. They show patterns and relative size or position, but it is more difficult to show 
causality. Viktor Mayer-Schönberger and Kenneth Cukier argue in their book Big Data (2013) that we no longer need causality. If we can predict how likely patients are to take their medicine based on their car insurance payment history, why would we want or need to know why they don't take their medicine, Mayer-Schönberger and Cukier ask. Obviously their payment history doesn't cause their tendency to take or not take medicines as prescribed. But it no longer matters. Causality for them is simply 'human intuiting' that doesn't deepen our insight, it is merely a 'cognitive shortcut that gives us the illusion of insight but in reality leaves us in the dark about the world around us' (2013, p. 64). Others are more concerned about the downplay of causality, as Wendy Chun writes: 'Big data [...] offers a form of cognitive mapping that allegedly sees all, by ignoring causes' (2017, p. 56).

Different forms of representation emphasize different relationships and patterns. Quetelet's data visualizations contributed to the idea of the average as something to be sought after, whereas earlier forms of representation, such as paintings, were well-suited to representing ideal beauty as something beyond human perfection. Data visualizations prioritize correlation. Narrative, by contrast, is a form of representation that often emphasizes causal connections. Narratives organize events in time. Some also provide causal connections between the events. E. M. Forster argues that such connections separate a story, which is just events in time ('and then, and then'), from a plot, which adds causality. "'The king died and then the queen died," is a story. "The king died, then the queen died of grief" is a plot,' Forster wrote (1949, p. 82). Roland Barthes, on the other hand, argued that 'the mainspring of narrative' is the reader's assumption that an event that happens after another event is caused by the first event, meaning that 'narrative would be a systematic application of the logical fallacy [...] post hoc, ergo propter hoc' (1977, p. 94). Causation is not always evident, but different forms of representation emphasize causation or correlation in different ways. Visualizations do not usually portray narratives, although this is certainly possible, as discussed by Wibke Weber and others in this volume.

One important extension of the correlation/causation binary is that the algorithmic processing of data that lies behind data visualizations often use proxies. Often, we cannot measure the things we are really interested in, so we find something that we can measure and that we assume has a direct relationship to the thing we actually want to understand. For instance, we don't have a way of directly measuring human emotions. Yet developers, or at least the marketers of their products, appear confident that using machine vision algorithms to analyse facial expressions can tell us that somebody is $99 \%$ angry and $0.5 \% \mathrm{sad}$, for instance. In this case, the facial expressions are 
proxies that are presumed to correlate perfectly with a person's emotions, although this assumption builds upon psychological theories that were arguably outdated decades ago (Bjørnsten \& Zacher Sørensen, 2017). We measure what we can measure and make claims based on that.

In other cases, maybe data could have been measured, but they were not, and so the datasets are incomplete. Machine learning can find correlations that appear to be valid in imperfect datasets. A useful example, discussed by economist Sendhil Mullainathan and medical researcher Ziad Obermeyer, demonstrates how machine learning in healthcare, despite excelling at 'predicting outcomes $y$ based on inputs $x$ ', can lead to misleading or biased predictions. This is because inputs such as medical records and insurance claim data suffer from 'large and systematic mismeasurement' (2017, p. 476). They give the example of predictors for having a stroke. It is often difficult to tell if patients arriving at a hospital are at risk of having a stroke, so a team used machine learning to analyse historical patient data in order to find factors in their medical history that are predictors of likelihood of having a stroke. On the surface, such a 'prediction problem' doesn't need to prove causal connections, since the goal is simply to plan for a more efficient use of resources, allocating more resources to patients with a higher risk of having a stroke. But although the machine learning algorithm had a lot of patient data, it did not have all the necessary data, because a lot of information about patients does not end up in their medical journals. The algorithm found that statistically valid predictors for having a stroke included having been treated for a minor injury due to a fall, or for acute sinusitis, or having had a scan for colon cancer. Upon closer inspection, human researchers found that the minor injuries and scans were in fact proxies for patients who were likely to go to the doctor for relatively minor issues. These patients were more likely than the general population to have a stroke diagnosed by a doctor, though not necessarily more likely to actually have a stroke, as many strokes are not diagnosed. Such skewed data can easily end up in well-intended data visualizations.

\section{Phantasmagrams and affect}

Sometimes, data visualizations are used to make predictive claims or arguments that can shape our understanding of the world. This can happen in a conceptual manner, as when Quetelet used data visualizations to develop the idea of the average as ideal, or in a more concrete way, as when a data series shows an increase or a decrease and the visualization suggests that this 
trend will continue into the future. This predictive use of data visualization is becoming more automated in systems such as those offered by Palantir and other companies for risk prediction. For instance, in predictive policing, police departments have a live map of their district with percentages and colour codes showing places where there is a high risk of certain crimes occurring, based on data analysis of past crimes as well as data such as local weather reports and the school calendar. When data visualizations make claims about the future, they can also affect the future, and we should be wary of how they do so.

Michelle Murphy has used the term phantasmagram to describe the way that 2oth-century economic and demographic models became not just descriptions of how the world works, but projections that lived lives of their own. She compares them to the phantasmagoria of the nineteenth century, 'ghostly simulations made by whirling magic lanterns that stimulated fright and awe' (Murphy, 2017, p. 53). She argues that demographic models are phantasmagrams, models that created new ways of seeing the world:

Through the work of Keynes and other similarly minded macroeconomists, the national economy was explicated as a new aggregate kind, a collective blur of activity that nonetheless could be modeled as a set of predictable correlations, tendencies, forces, and rates representable in equations and graphs. When interest rates go up, investment goes down, employment drops, output falls. With equations and diagrams, mathematical modelling in the 193os performatively discerned 'the economy' as a constellation of such interrelationships within a closed system whose boundary was the nation-state. (Murphy, 2017, p. 18)

The very idea of it being possible to measure the entire economic performance of a country as its Gross Domestic Product (GDP) is a phantasmagram, Murphy argues, which will always leave things out (unpaid labour, for instance) and miscount other components. GDP is an example of 'quantitative practices that are enriched with affect, propagate imaginaries, lure feeling, and hence have supernatural effects in surplus of their rational precepts' (2017, p. 24). The success of such a model lay 'not in its empirical veracity but in the way it gave form to a technocratic dream of a national macroeconomy that could be fostered, directed, and triggered by rearranging reproduction en mass,' Murphy argues (2017, p. 51).

We are used to thinking of quantitative models or visualizations as objective and rational. This is what José van Dijck calls dataism, as noted above. For Murphy to instead highlight the affect and even the sublime 
of economic models (2017, pp. 9, 23) is a very different approach that may seem at odds with our everyday experience of models, graphs, and other data visualizations. Perhaps, as Helen Kennedy and Rosemary Hill note, it is the very combination of the 'statistical and visual' in data visualizations that leads to their emotional impact (2017, p. 831). Kennedy and Hill discuss a range of emotional responses that participants in their focus groups expressed when looking at data visualizations. Here, I will discuss the sense of the sublime that Murphy touches upon.

The sublime is an old concept, used first by Longinus around 2000 years ago. For Longinus, the sublime was a rhetorical technique used in a speech to 'overcome the rational powers' of an audience (Longinus, 100 $\mathrm{CE} / 1935)$. While Longinus theorized the sublime as a rhetorical technique for influencing people, Kant saw it as a human response to grandeur in art or nature. His concept of the mathematical sublime is awakened in us when we sense something that is absolutely large: it isn't of a specific size, it is great without comparison, so we can't grasp it mathematically. The sublime, for Kant, lies not in the object but in our experience of it. If you gaze at the night skies, or consider undying love or loyalty, then you may experience the sublime. Combining Kant and Murphy's ideas, then, we might say that the vastness of the idea of GDP, of being able to compute and visualize all the economy of all the world, also awakens this sense of the sublime.

The pleasure of the sublime, Kant writes, lies in the sense that our mind is broadened by this experience of the infinite that allows us to 'pass beyond the narrow confines of sensibility' (2007, p. 256). This sounds close to the reaction that designer Jer Thorp says he aims for when he designs a data visualization: 'First, it needs to be visually pleasing. I want people to say 'Oooh....' when they turn the page to it. Once they're hooked, though, I want them to learn something — the 'Aaah!' moment' (2010). The initial pleasure should give way to rational understanding. Although some have criticized the obsession with the visual beauty of data visualizations (McCosker \& Wilken, 2014), Kant's idea of the sublime as something that can lead to a deeper understanding can also be seen as aligned with ideas of embodied knowledge and the role of emotions and the senses in knowledge. Affect and emotion can offer us kinds of knowledge that are not directly accessible through purely rational analysis.

Data visualizations are not simply visual and they are not simply quantitative. They are a form of communication that emphasizes data. Sometimes it is the very fact that they present reality as understandable and predictable through data models that makes data visualizations so convincing. 


\section{References}

Barthes, R. (1977). Image music text. (S. Heath, Trans.). London: Fontana Press.

Bjørnsten, T. B., \& Zacher Sørensen, M.-M. (2017). Uncertainties of facial emotion recognition technologies and the automation of emotional labour. Digital Creativity, 28(4), 297-307. https://doi.org/10.1080/14626268.2017.1383271

Chun, W. H. K. (2017). Updating to remain the same: Habitual new media. Cambridge, MA: MIT Press.

Davis, L. J. (2013). Introduction: Disability, normality, and power. In: L. J. Davis (Ed.), The disability studies reader. (pp. 1-17). Florence, KY: Taylor \& Francis.

D’Ignazio, C., \& Klein, L. (2016, October). Feminist Data Visualization. Paper presented at and published in the workshop proceedings from the Workshop on Visualization for the Digital Humanities at IEEE VIS Conference, Baltimore, Maryland.

Drucker, J. (2011). Humanities approaches to graphical display. Digital Humanities Quarterly, 5(1). Retrieved June 12, 2019 from http://www.digitalhumanities.org/ $\mathrm{dhq} / \mathrm{vol} / 5 / 1 /$ oooog1/oooog1.html

Drucker, J. (2014). Graphesis: Visual forms of knowledge production. Cambridge, MA: Harvard University Press.

Eubanks, V. (2018). Automating inequality: How high-tech tools profile, police, and punish the poor. New York: St. Martin's Press.

Forster, E. M. (1949 [orig. 1927]). Aspects of the novel (Pocket ed.). London: Edward Arnold \& Co.

Friendly, M. (2008). A brief history of data visualization. In: C.-H. Chen, W. Härdle, \& A. Unwin (Eds.), Handbook of data visualization. (pp. 15-56). Berlin: Springer.

Gates, K. (2011). Our biometric future: Facial recognition technology and the culture of surveillance. New York: NYU Press.

Gillespie, T., \& Seaver, N. (2015, November 5). Critical Algorithm Studies: a Reading List [Blog post]. Retrieved from https://socialmediacollective.org/reading-lists/ critical-algorithm-studies/

Gitelman, L. (Ed.). (2013). Raw data is an oxymoron. Cambridge, MA: MIT Press.

Haraway, D. (1988). Situated knowledges: The science question in feminism and the privilege of partial perspective. Feminist Studies, 14(3), 575-599. https://doi. org/10.2307/3178066

Hill, R. L., Kennedy, H., \& Gerrard, Y. (2016). Visualizing junk: Big data visualizations and the need for feminist data studies. Journal of Communication Inquiry, 4o(4), 331-35o. https://doi.org/10.1177/0196859916666041

Kant, I. (2007). Critique ofjudgement (J. C. Meredith, Trans.; N. Walker, Ed.). Oxford \& New York: Oxford University Press.

Kember, S. (2014). Face recognition and the emergence of smart photography. Journal of Visual Culture, 13(2), 182-199. https://doi.org/10.1177/1470412914541767 
Kennedy, H. (2016). Post, mine, repeat: Social media data mining becomes ordinary. London: Palgrave Macmillan Limited.

Kennedy, H., \& Hill, R. L. (2017). The feeling of numbers: Emotions in everyday engagements with data and their visualisation. Sociology, 52(4), 830-848. https:// doi.org/10.1177/0038038516674675

Leaver, T. (2017). Intimate surveillance: Normalizing parental monitoring and mediation of infants online. Social Media + Society, 3(2). https://doi. org/10.1177/2056305117707192

Longinus. (1935 [ca. $100 \mathrm{CE}])$. On the sublime (W. R. Roberts, Trans.) Cambridge: Cambridge University Press.

Mackenzie, D. (1993). Inventing accuracy: A historical sociology of nuclear missile guidance. Cambridge, MA: MIT Press.

Mayer-Schönberger, V., \& Cukier, K. (2013). Big data: A revolution that will transform how we live, work, and think. Boston, MA: Houghton Mifflin Harcourt.

McCosker, A., \& Wilken, R. (2014). Rethinking 'big data' as visual knowledge: The sublime and the diagrammatic in data visualisation. Visual Studies, 29(2), 155-164. https://doi.org/10.1080/1472586X.2014.887268

Mullainathan, S., \& Obermeyer, Z. (2017). Does machine learning automate moral hazard and error? American Economic Review, 107 (5), 476-48o. https:// doi.org/10.1257/aer.p20171084

Murphy, M. (2017). The economization of life. Durham, NC: Duke University Press.

Noble, S. U. (2018). Algorithms of oppression: How search engines reinforce racism. New York: NYU Press.

Olson, P. (2018, June 28). This AI just beat human doctors on a clinical exam. Forbes. Retrieved from https:/www.forbes.com/sites/parmyolson/2018/06/28/ ai-doctors-exam-babylon-health/

O'Neil, C. (2017). Weapons of math destruction: How big data increases inequality and threatens democracy. London: Penguin.

Ong, W. J. (1982). Orality and literacy: The technologizing of the word. London: Routledge.

Oppenheimer, G. (2013). Medical care of infants in the United States: A review of recent work. In: R. Greene (Ed.), History ofmedicine. (pp. 103-128). Florence, KY: Taylor and Francis.

Quetelet, A. (1997). Physique sociale ou Essai sur le développement des facultés de l'homme [1869]. (É. Vilquin \& J.-P. Sanderson, Eds.). Brussels: Académie Royale de Belgique.

Rettberg, J. W. (2014). Seeing ourselves through technology: How we use selfies, blogs and wearable devices to see and shape ourselves. Basingstoke: Palgrave.

Sekula, A. (1986). The body and the archive. October, 39, 3-64. https://doi. org/10.2307/778312 
Sterne, J. (2011). The theology of sound: A critique of orality. Canadian Journal of Communication, $36(2)$. https://doi.org/10.22230/cjc.2011v36n2a2223

Tal, A., \& Wansink, B. (2016). Blinded with science: Trivial graphs and formulas increase ad persuasiveness and belief in product efficacy. Public Understanding of Science, 25(1), 117-125.

Thorp, J. (2010, July 12). Wired UK, Barabási Lab and Big Data [Blog post]. Retrieved from http://blog.blprnt.com/blog/blprnt/wired-uk-barabasi-lab-and-big-data Tversky, B. (2010). Visualizing thought. Topics in Cognitive Science, 3(3), 499-535. https://doi.org/10.1111/j.1756-8765.2010.01113.x

van Dijck, J. (2014). Datafication, dataism and dataveillance: Big Data between scientific paradigm and ideology. Surveillance \& Society, 12(2), 197-208. http:// doi.org/10.24908/ss.v12i2.4776

Wachter-Boettcher, S. (2017). Technically wrong: Sexist apps, biased algorithms, and other threats of toxic tech. New York: Norton.

\section{About the author}

Jill Walker Rettberg is Professor of Digital Culture at the University of Bergen, and principal investigator of an ERC (European Research Council) project that explores how machine vision and new algorithmic images are affecting us as a society and as individuals. She is the author of Blogging (Polity, 2014) and Seeing Ourselves Through Technology (Palgrave, 2014). 